\title{
Improving Mouse Pointing for Radiology Tasks
}

\author{
Yan Tan, Geoffrey Tien, Bruce Forster, M. Stella Atkins \\ Simon Fraser University \\ University of British Columbia
}

\begin{abstract}
Radiologists make their main analysis and diagnosis based on careful observation of medical images, although there are all kinds of automatic methods under development. Radiologists typically use a scroll mouse to click on an image when they find something interesting, and they also use the mouse to navigate through the image slices in volumetric scans. Thus they perform many thousands of mouse clicks every day, causing wrist fatigue. This paper presents a method of improving the mouse pointing performance by reducing the time taken to move the mouse to a target. We use a dynamic Control-to-Display ratio of the mouse, by adjusting the ratio according to the current distance to the target. In theory this reduces the difficulty of the target selection, and also reduces the movement time. The result of a preliminary study demonstrates that the speed of pointing can be improved under certain conditions, particularly for small targets and for long distances to move. In addition, all participants claim that this mouse speed change reduces the difficulty of selecting a small target.
\end{abstract}

Keywords: workstation design, human interaction, CAD development, observer study

\section{INTRODUCTION}

In order for image viewing software and user interaction hardware to be valuable, it must display the images in a manner that is useful for the user tasks. Pointing and clicking at targets on images are very common tasks for radiologists. When an interesting "target" is found, it may be necessary to click on that target, usually requiring a mouse movement and click. Typically, when MRI and CT images have an axial and a coronal view displayed on two monitors, it is necessary to repeatedly move the cursor from one monitor to another for crossreferencing. Additionally, scrolling through 2-D slice sequences of 3-D volume data typically requires a lot of mouse movement. ${ }^{1}$ It is easy to become fatigued and stressed after hundreds of such procedures, particularly when the target place to click is small, and the distance to move is large, such as across two display monitors. ${ }^{2}$ The goal of this research is to improve the performance of such target selection.

We take advantage of the mouse control-to-display (C-D) gain ratio which is the ratio of the distance the mouse moves in physical (motor) space, to the corresponding distance the cursor moves on the display. With a low C-D gain, the cursor moves more slowly, and by increasing the C-D gain, the same movement in hand space corresponds to a larger movement of the cursor on the screen. Now Fitts' Law ${ }^{3}$ states that the index of difficulty in pointing to a target, is proportional to the logarithm of (distance/size). Thus to reduce the index of difficulty and improve performance for pointing tasks, we need to dynamically change the mouse speed according to the distance to the target.

Fitts' law has shown that as the target becomes smaller, the index of difficulty increases. ${ }^{3}$ A movement task's difficulty, the Index of difficulty (ID) can be quantified using information theory using the metric bits where $I D=\log _{2} \frac{2 D}{W}$ where $D$ is the distance or amplitude to move and $W$ is the width or tolerance of the region within which the move terminates. This applies to one-dimensional pointing tasks. By experiment, researchers found the average movement time has a linear relationship with the $I D$. To be generalized into two or three dimensions, other issues such as the shape of targets need to be considered.

From the formula: $I D=\log _{2} \frac{2 D}{W}$, it can be seen that the distance to the target is another factor that affects the movement time. As the resolution and size of modern displays are much larger than before, the number of pixels in one dimension has increased. Thus the visual distance and motor distance (under a constant C-D

Further author information: (Send correspondence to Yan Tan, Geoffrey Tien): E-mail: yta19@cs.sfu.ca gtien@cs.sfu.ca

Medical Imaging 2009: Image Perception, Observer Performance, and Technology Assessment, edited by Berkman Sahiner, David J. Manning, Proc. of SPIE Vol. 7263, 72631I

(C) 2009 SPIE $\cdot$ CCC code: $1605-7422 / 09 / \$ 18 \cdot$ doi: $10.1117 / 12.812278$

Proc. of SPIE Vol. 7263 72631I-1 
gain) of screens is longer than before. Obviously, increasing the mouse movement speed (increasing the C-D gain) can reduce the motor distance to the target. However, researchers found that there is a constraint of the speed-accuracy trade-off. ${ }^{4}$ That is, as the speed goes up, the error rate increases in the meantime. Another implication of reducing the ID by increasing the speed is the reduced relative width of targets. When the C-D gain is increased to achieve a faster cursor speed, the relative width of a target is effectively reduced. Therefore, it is not always possible to improve pointing performance by merely increasing the device's moving speed.

Previous work has shown that dynamically adjusting the C-D ratio can efficiently enhance pointing performance. $^{5-7}$ Most of these works are based on One-Dimensional experiment. Blanch et al ${ }^{8}$ have shown that slowing down the mouse when it is over the target area helps improve pointing performance. When applied to two-dimensions, the situation becomes more complicated. In some scenarios where the target area is very small, the effect of this technique is limited because visually locating the target is more difficult. Furthermore, this technique relies on the prediction of target position. Some target prediction methods have been developed, with most of them based on Graphical User Interface (GUI) components. ${ }^{9,10}$ However, when there is no GUI component on the target area such as when pointing or marking on a large bitmap, it is hard to predict where the user intends to point. In such cases, we propose that an eye-gaze tracking system may be used for target prediction since researchers have found that people look at what they are working on. ${ }^{11}$

This paper proposes a method based on the dynamic C-D ratio, to improve the speed and precision of mouse tasks in radiology workstations.

\section{DYNAMIC CONTROL-TO-DISPLAY RATIO 2.1 DYNAMIC CONTROL-TO-DISPLAY IN 1-D SPACE}

The index of difficulty can be reduced by expanding the target size in visual space or motor space. Because the $\mathrm{C}-\mathrm{D}$ ratio defines the distance that the mouse has to cover in the physical world (motor space), this paper presents a dynamic C-D ratio which decreases the C-D gain when the cursor is close to the target, thus expanding the target size in motor space. As the C-D gain decreases, crossing the same number of pixels on the screen requires more movement in motor space, enlarging the motor width of the target. This method has been shown to improve pointing performance..$^{5,8}$

More specifically, by decreasing the C-D gain while approaching targets, the width of the target becomes effectively bigger in motor space. When the cursor is not close to the target, the C-D ratio remains at the initial value. Therefore, the $W$ (width) in Fitts' law increases while the $D$ (distance) remains, reducing the index of difficulty.

As can been seen in equation 2 , once mouse movement speed is reduced by dividing by the slowing down ratio $R$, the effective width becomes $W R$. Additionally, the space in which the cursor movement speed is reduced will be called the slowing down area. If the cursor slows down just over the target, then the $I D$ is reduced by subtracting $\log _{2} R$, as shown in equation 2. Theoretically, the greater $R$ becomes, the more $I D$ is decreased.

$$
\begin{aligned}
I D & =\log _{2} \frac{2 D}{W R}, \text { where } \mathrm{R} \text { is the slowing down ratio } \\
& =\log _{2} \frac{2 D}{W}-\log _{2} R
\end{aligned}
$$

\subsection{DYNAMIC CONTROL-TO-DISPLAY RATIO IN 2-D SPACE}

Although the dynamic C-D ratio is proved to be effective to improve mouse performance, most of the studies were conducted in 1-D space. The advantage of choosing 1-D space is that the target width is strictly defined. Also, in 1-D space, it is very easy to determine whether the cursor is moving towards or away from the target.

In 2-D space, the situation becomes more complicated. The first challenge for 2-D space is that the target width is much harder to determine due to different target shapes and entry angles. Several methods have been proposed to solve this problem. One is called "STATUS QUO", ${ }^{12}$ which uses the horizontal extent of the target. A second and more sophisticated method is to substitute for $W$ a measure more consistent with the 2-D nature 


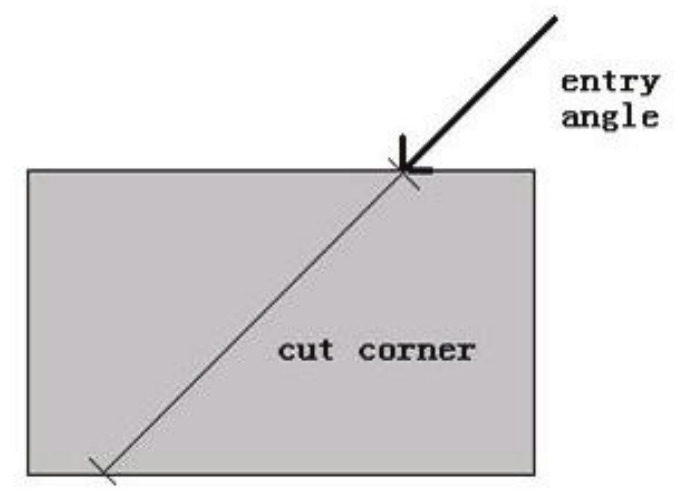

Figure 1. Using "cut corner" as target width

of the task. It uses the "cut corners" (figure 1) as the target width"12. Another possible method is "the smaller of $W$ or $H "$. In this method, the target width is defined as the smaller of the two dimensions.

Path variation is another issue brought to 2-D space. In 1-D tasks, there is only one path from the starting point to the target. This important feature makes the dynamic C-D ratio very effective in 1-D. In this case, the greater $R$ is, the less is $I D$. In 2-D tasks, path variations should be considered. Target re-entry, task axis crossing, movement direction change and orthogonal direction change can significantly affect the movement time. ${ }^{13}$ Therefore, only increasing the value of $R$ cannot always reduce value of $I D$ especially when targets are small.

We propose that if we are able to expand the slowing down area introduced in section 2.1 beyond the visual boundaries of the target, the influence of path variations can be reduced since the mouse cursor is relatively easily captured. In addition, the expanded slowing down area is able to tolerate inaccurate target prediction or acquisition, making the implementation more practical. For example, in some situations, potential targets are located close to each other, or there is no way to predict targets at all, the actual target can still be covered by the expanded slowing down area of the predicted target.

If we define $D^{\prime}$ as the new effective distance, $W^{\prime}$ as the new effective target width, $S$ as the slowing down area and $C D$ as the $\mathrm{C}-\mathrm{D}$ ratio, then

$$
\begin{aligned}
D^{\prime} & =\int_{0}^{D} C D(x) d x \\
& =\left(D-\frac{S}{2}\right) \times C D+\frac{S}{2} \times C D \times R \\
W^{\prime} & =\int_{0}^{W} C D(x) d x=W \times C D \times R \\
I D & =\log _{2} \frac{2 D^{\prime}}{W^{\prime}} \\
& =\log _{2} \frac{2 \times\left(\left(D-\frac{S}{2}\right)+\frac{S}{2} \times R\right)}{W \times R}
\end{aligned}
$$

Equations 4 and 5 show that the new effective distance $D^{\prime}$ and new effective width $W^{\prime}$ increase together. Equation 7 recalculate the ID based on the $D^{\prime}$ and $W^{\prime}$. Although we add some extra distance to move in motor space, it does not significantly affect the $I D$ when the $D$ is large and $W$ is small. 


\subsection{Implementation of dynamically adjusted C-D ratio}

We use the Windows XP Application Programming Interface (API) to dynamically adjust the C-D ratio during the movement. By capturing the mouse movement events, we set the C-D ratio according to the current position of the mouse cursor. However, in Windows XP, the built-in "acceleration" has been added as a parameter for mouse movement, that is, when the speed of the mouse reaches a certain threshold, the C-D ratio will be changed automatically. In order to control the C-D gain during the experiment, we remove the built-in "acceleration" during the experiment.

There are 20 levels of mouse speed (from 1 to 20). By experiment we found that the C-D gain is linearly changed from the lowest to the highest. Thus, the new cursor movement speed $V^{\prime}$ under slowing down ratio $R$ could be $V^{\prime}=\frac{V}{R}$ where $V$ is the current mouse speed. Correspondingly, the new C-D gain $C D^{\prime}$ is $C D^{\prime}=\frac{C D}{R}$ where $C D$ is the current $\mathrm{C}-\mathrm{D}$ ratio.

\section{METHODOLOGY}

In order to evaluate the performance improvement of this new dynamic C-D ratio technique, we designed a series of experiments. All experiments were conducted following a strict written procedure. The whole experimental procedure was managed by the experiment administrator. Subjects were first introduced to the mouse tasks, and completed a general questionnaire to gain information regarding their age, education and experience of using a mouse.

Before performing the full study that evaluated the performance improvement of our method, we firstly conducted a pilot study to determine the best parameter combination for $S$ and $R$.

\subsection{PILOT STUDY}

In our new dynamic C-D ratio method, we expanded the slowing down area $S$. However, not only does increasing $R$ enlarge the effective width of targets, but it also affects $D^{\prime}$. Since $S$ is much bigger than the target width, we can no longer ignore the impact that a larger $R$ brings to $D^{\prime}$. Therefore, we present a pilot study to evaluate how users' performance changed in performing a pointing task to a known target. We adjusted both size of the slowing down area, and the slowing down ratio of the known targets to ascertain how much the pointing time changed, by decreasing the C-D gain dynamically.

Three graduate students with abundant experience in mouse clicking participated in the pilot study. For each subject, there were 15 parameter combinations, with each combination consisting of 3 target distances. For each target distance, there were four tasks with the same distance but different positions (random).

According to the result from pilot study, we have found that the parameter combination of $R=2$ and $S=80$ pixels gives us the best performance improvement, so we chose this parameter combination for the full study.

\subsection{FULL STUDY}

The full study consisted of two experiments. 12 students from Simon Fraser University (average age 24.8) were recruited, all are experienced mouse users. The first experiment was to evaluate the performance gain for small targets (red dots each with a width of 10 pixels) under dynamic C-D ratio. The experimental platform is shown in figure 2. In this experiment there were four image boxes with each showing a MRI image. Basically, subjects were required to target dots on those images in a specified order using a mouse. To begin the experiment, subjects first right-clicked anywhere on the screen to activate a popup menu, and selected "linking" from the menu. Next they clicked on each red dot as they appeared; there were 8 targets spread among each set of four images. When all 8 dots had been clicked, subjects again right-clicked and selected "linking" to begin the next set. In one block, each group of four images was used four times. Then the total number of mouse tasks (completion of clicking a specified object) is 8 dots $\times 4$ sets $\times 4$ blocks $=128$ dots clicks plus $4 \times 4=16$ for right-clicks and button clicks.

The second experiment aimed to include a larger variety of tasks commonly encountered in radiologists' daily working environment. 4 subjects who had participated in the first experiment were recruited for the second experiment (average age: 25.3). This experiment consisted of tasks of dragging and dropping image thumbnails from a vertical strip to image boxes, and targeting dots on each image. In this experiment, four sizes of targets 


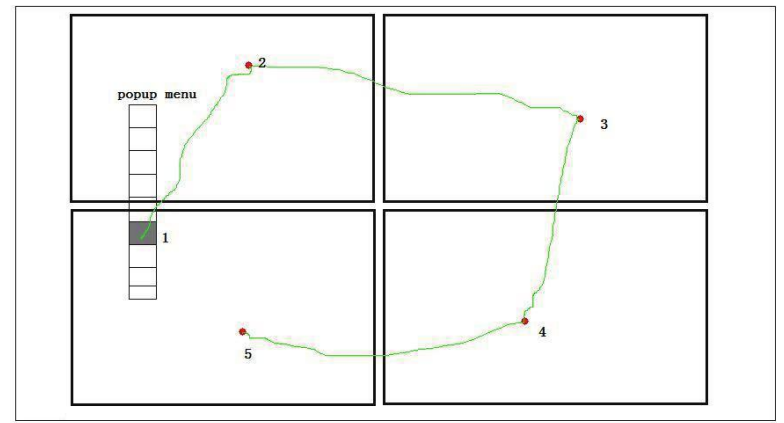

Figure 2. Experiment platform layout for small tasks

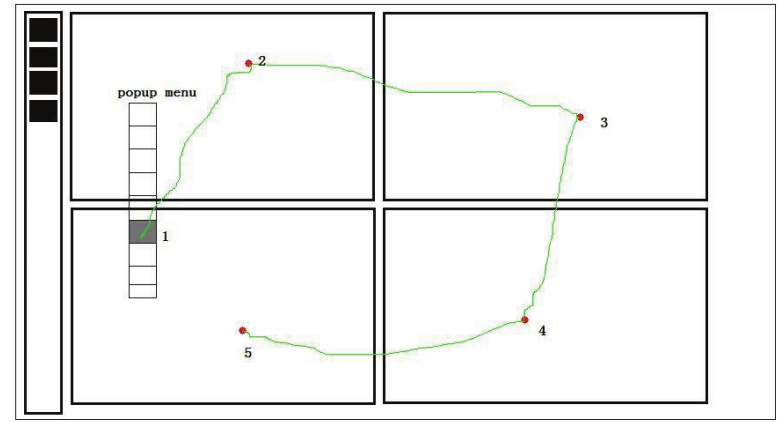

Figure 3. Experiment platform layout for simulating radiologist's tasks

were involved which are image thumbnails, image boxes, the context menu buttons, and dots on each image with each size of $140 \times 140,500 \times 500,200 \times 50$ and $10 \times 10$ pixels respectively. The total number of tasks in this experiment is $4 \times 4 \times 4=64$ for dragging and dropping tasks, $4 \times 4=16$ for right-click and button clicks and $8 \times 4 \times 4=128$ for dots clicks.

The experimental platform is shown in figure 3. In this experiment, subjects first dragged and dropped a thumbnail to a specified image box. In order to reduce the reaction time, when subjects dragged on thumbnails, only the corresponding image box remained on the screen, while the others were hidden. After the four thumbnails had been dragged into their respective image boxes, subjects right-clicked to bring the popup menu to the screen, and then left-clicked the specified button to display the first dot on one of the four images. Then they performed the targeting for eight dots on the screen in order. This whole procedure is called one set. Again, in one block, each group of four images was used five times with different dragging and dropping order. Therefore, there were 4 sets * 4 blocks (within each block the same four images are used).

To maintain consistency across experimental blocks, it is important to consider the order in which the various tasks are performed. First, the order must be determined of the four image thumbnails to be dragged and dropped in the second experiment. As required, subjects always started from the topmost thumbnail and performed the rest downwards. In different sets, thumbnails were forced to different image boxes. These orderings were arranged pseudo-randomly (pre-set) and are shown in figure 4.

The second consideration is of the order in which the eight small dots must be clicked. If dots appear on the screen in a fixed order, subjects might easily predict the location of the next target, and this could affect the generality of our results. Also, appropriate arrangement of dots order can provide us more effective data, enabling us to evaluate different distances and angles. In our experiment, the angle 0 degrees was defined as that horizontally from the left to the right. A clockwise change corresponded to an increase in angle. In our experiment, there were four sets of tasks in each block. Distances for each set, with angle of 90 and 270 were 


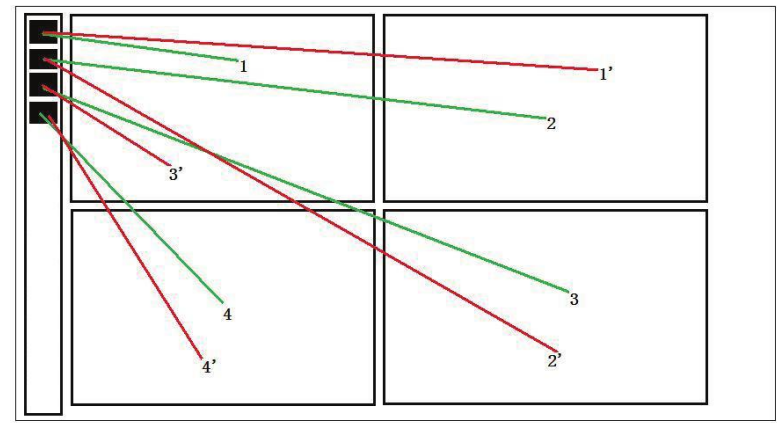

Figure 4. Experiment platform layout for small tasks

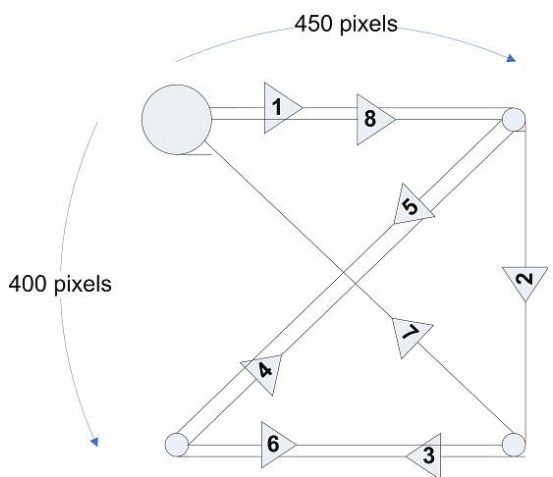

(a)

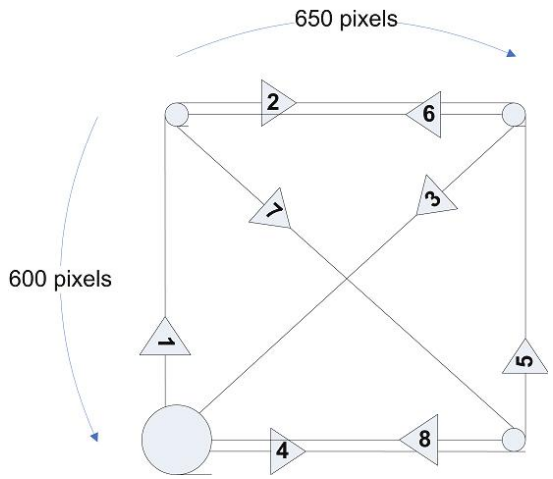

(c)

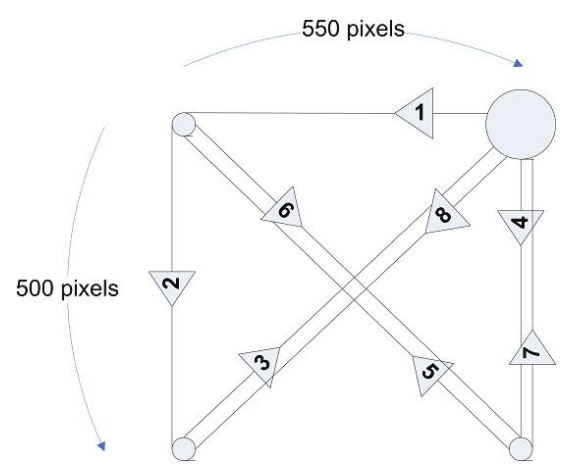

(b)

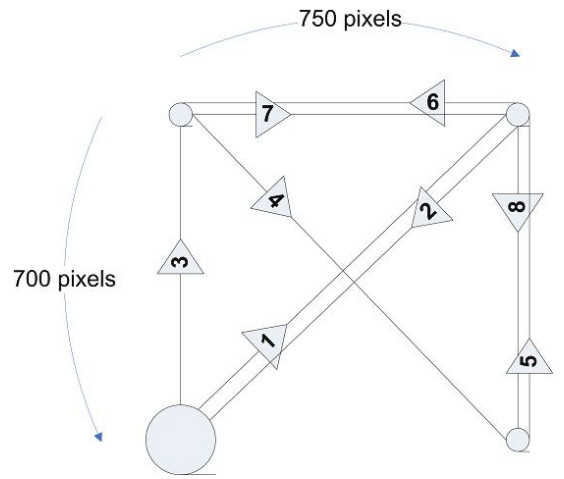

(d)

Figure 5. The order in which targets appear on the screen. The big circle indicates the starting point, however, subjects may click other place to activate "linking"

400, 500, 600 and 700. Distances with angle of 0 and 180 were 450, 550, 650 and 750. Distances with angle of $45,135,225,315$ were $600,750,884$ and 1025. Dots sequences for each set are shown in figure 5 . We applied this sequence to all four blocks.

For each experiment, all tasks were performed under two conditions (constant C-D ratio and dynamic C-D ratio). In order to minimize the impact of fatigue, subjects were required to have a short break every five minutes. 
Also, for statistical validity, the order in which subjects completed each condition was counterbalanced, with half of the subjects performing the tasks under constant C-D ratio before the dynamic C-D, and vice-versa for the other half of subjects. After each condition, subjects were requested to complete a questionnaire to report their thoughts about the preceding condition. After completion of both conditions, each subject was given a questionnaire for subjectively comparing any perceived difference between the two conditions.

The initial mouse speed was set to 10 (windows scales mouse speed from 1-20 where 1 represents the slowest and 10 is the default setting). The slowing down ratio we used is $R=2$ for dots and 3.3 for other targets (thumbnails and image boxes). The slowing down area was $S=80$ pixels in diameter for all targets. Currently we are more interested in the performance improvement on dots clicks. The expected ID (index of difficulty) for different distances is shown in table 1.

Table 1. Original ID vs new ID where the new ID $I D^{\prime}=\log _{2} \frac{2 \times\left(\left(D-\frac{S}{2}\right)+\frac{S}{2} \times R\right)}{W \times R}, R=2, S=80$

\begin{tabular}{r|r|r|r}
\hline $\mathrm{D}$ (pixel) & W (pixel) & original ID (bit) & new ID (bit) \\
\hline 400 & 10 & 6.32 & 5.39 \\
500 & 10 & 6.64 & 5.7 \\
565 & 10 & 6.82 & 5.87 \\
600 & 10 & 6.91 & 5.95 \\
650 & 10 & 7.02 & 6.07 \\
700 & 10 & 7.13 & 6.17 \\
707 & 10 & 7.14 & 6.18 \\
848 & 10 & 7.41 & 6.44 \\
989 & 10 & 7.63 & 6.66 \\
\hline & &
\end{tabular}

Throughout the table 1, we predict that the improved dynamic C-D ratio can bring us an average of $13.6 \%$ performance improvement since the movement time is linearly corresponding to the $I D .^{3}$

During the experiment, every single mouse click was recorded.

\section{RESULT AND DISCUSSION}

For the experiment for small targets, since there was no training stage for subjects, we needed to firstly recognize the trials during which subjects were still becoming accustomed to the task and cursor speed. The figure 4 shows the total completion time for each block under constant C-D ratio and dynamic C-D ratio. We have observed that out of the four blocks in the overall procedure, the first block has the longest completion time under both constant C-D ratio and dynamic C-D ratio. Additionally, the remaining 3 blocks have a relatively stable performance. This result enables us to conservatively assume that the duration of tasks in the first block was enough for subject to adapt to the experimental platform and mouse movement tasks. Furthermore, figure 7 shows the performance difference between training stage (the first block) and the remaining three blocks. We can see that in both constant C-D ratio and dynamic C-D ratio, there is a gap between the training stage and the remaining three blocks. This confirms the assumption that the subjects had already adapted to the experimental setup after the first block.

As described above, we can remove learning effects and evaluate the performance improvement by analyzing the remaining 3 blocks in the data set. From figure $8(\mathrm{a})$ which shows the average movement time and standard deviation corresponding to distances under constant C-D ratio and dynamic C-D ratio, we can see that the average movement time under dynamic C-D ratio is always shorter than that under constant C-D ratio for all distances. The p-value of the difference between constant and dynamic C-D ratio is shown in figure $8(\mathrm{~b})$. While the differences are not all significant at the 0.01 level, the weighted average of the p-values across all distances is significant at $p<0.01$. Figure 9 shows the improvement percentage. The highest improvement is $19.21 \%$ which occurs at the distance of 450 pixels, while the lowest is $11.74 \%$ which occurs at the distance of 700 pixels. This 


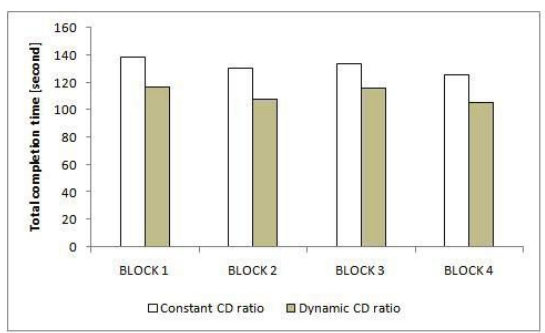

Figure 6. The total completion time for each block.

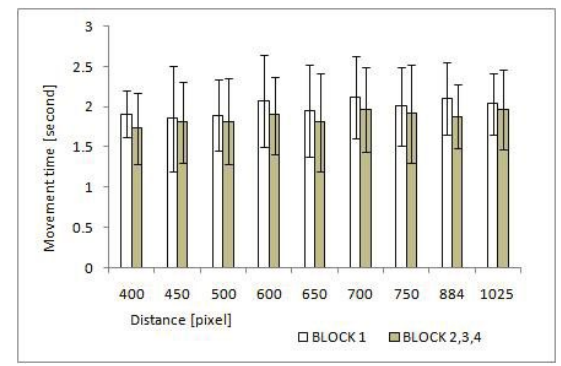

(a)

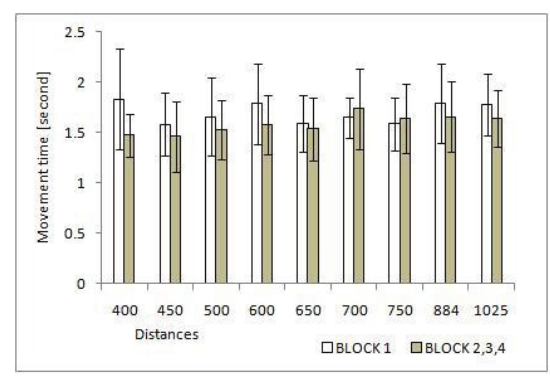

(b)

Figure 7. The average movement time and standard deviation corresponding to distances. First block vs the remaining three blocks. The left figure is that under constant C-D ratio, the right figure is that under dynamic C-D ratio

result is consistent with our prediction based on table 1 that the improved dynamic C-D ratio would provide $15 \%$ performance improvement.

By looking at the standard deviation in figure 8(a), we have found that the standard deviation for each distance is not minor. This can be explained by that different subjects have different familiarity of mouse. Also, by looking into the questionnaire, we have found that some subjects are left-handed mouse users. These left-handed users would certainly perform less accurately using the right-handed mouse, and spend more time on each trial. Some other people complained that the default mouse speed was not what they were familiar with, and claimed that they could perform much better under their own preferred mouse speed. However, all our subjects were required to perform under the same experimental settings for generality, leading us to use the first experimental block as practice for each subject.

With respect to pointing accuracy, the dynamic C-D ratio always provided a lower error rate compared to constant $\mathrm{C}-\mathrm{D}$ ratio, even during the training period (figure 10). The result is consistent to the questionnaire

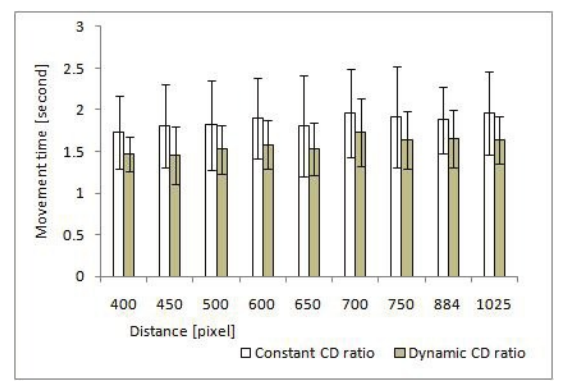

(a)

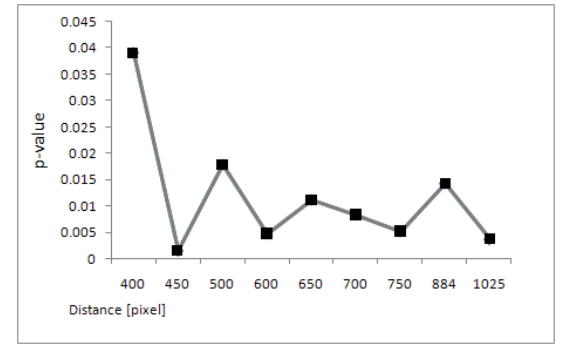

(b)

Figure 8. a)The average movement time and standard deviation corresponding to distances. Constant C-D ratio vs Dynamic C-D ratio. b)The p-value of significance of difference for each distance. 


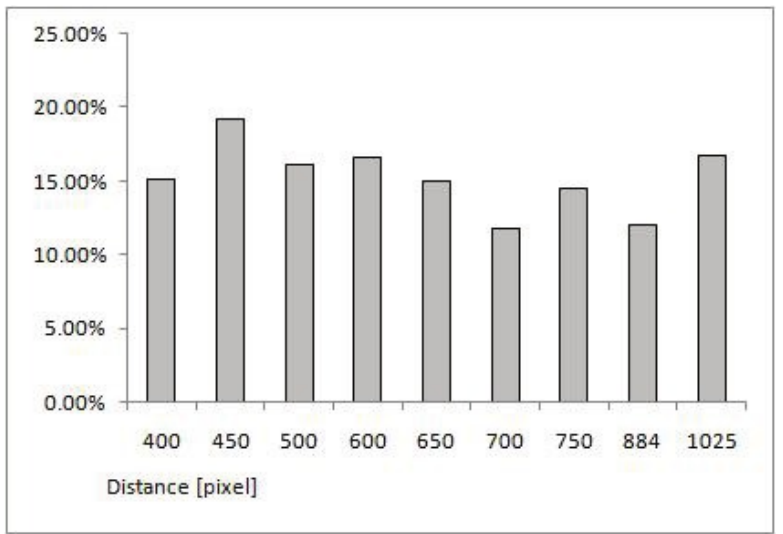

Figure 9. The performance improvement percentage from dynamic C-D ratio corresponding to distances

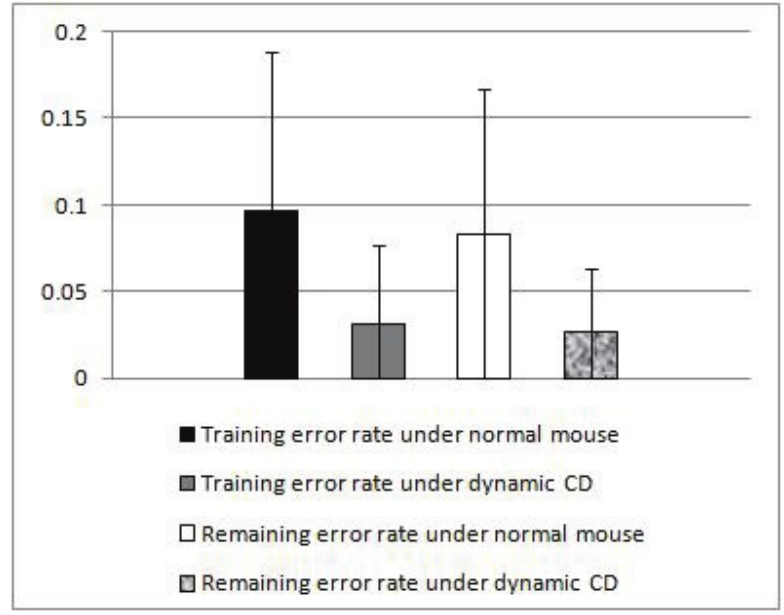

Figure 10. The error rate.

that all subjects claim they could perform equally or more accurately under the dynamic C-D ratio than that under the constance $\mathrm{C}-\mathrm{D}$ ratio.

For the perceptivity, more than $\frac{2}{3}$ of subjects claimed they did not feel any difference during the experiment. Of the remaining $\frac{1}{3}$ of subjects who did notice a difference, all preferred the dynamic C-D ratio. Of interest is one subject who did not feel any difference but still ranked the dynamic C-D ratio above the constant C-D ratio. No subjects preferred the constant C-D ratio.

Finally, we evaluate the result of the second experiment which simulated the real radiologists' working environment. Figure 11 shows the total completion time for all subjects. All subjects performed slightly faster under dynamic C-D ratio. Figure 12 shows the average error rate and standard deviation relative to both clicking on dots and thumbnails under constant C-D ratio and dynamic C-D ratio. As can be seen, the average error rate is lower for both clicking on dots and thumbnails under dynamic C-D ratio.

\section{CONCLUSION}

Comparing to the normal dynamic C-D ratio method, the improved dynamic C-D ratio method expands the slowing-down area, making it easier to capture the mouse cursor. Based on the parameter we found in pilot study, 


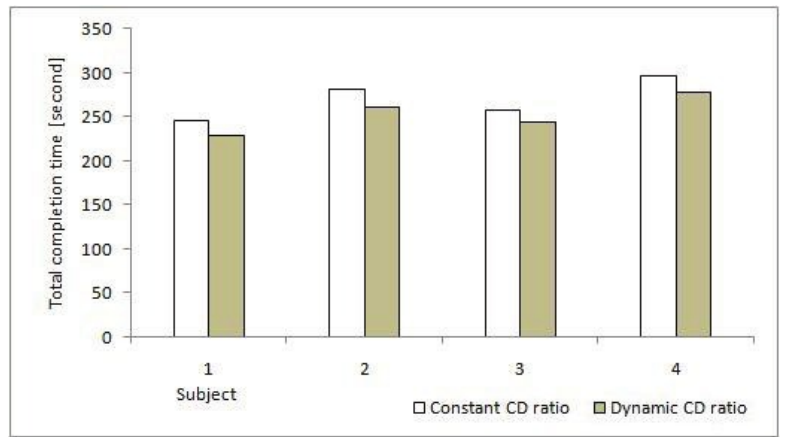

Figure 11. The total completion time for simulating the real radiologist's working environment by each subjects.

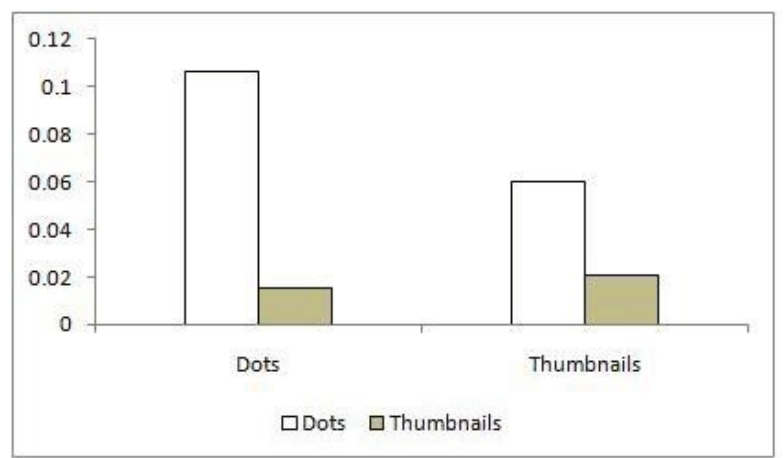

Figure 12. The average error rate and standard deviation for simulating the real radiologist's working environment

our implementation indicates that this method can bring an average of $15 \%$ performance improvement, with a lower error rate and user preference for clicking on small targets. Our experiment also shows that combined with regular radiologist's tasks such as dragging and dropping thumbnails and clicking buttons, our method brings a shorter total completion time comparing to that under constant C-D ratio, again, with a lower error rate.

Currently, all targets are preset (known). In the future, we plan to employ eye-gaze tracking for target prediction, which can enable us to generalize our method to all kinds of mouse tasks without any knowledge of the underlying interface or tasks. Our preliminary work using eye-gaze tracking is very promising ${ }^{14}$. Additionally, we may need to enhance our method by only slowing the cursor down when it is approaching the target. Furthermore, we might evaluate the potential performance improvement from accelerating the mouse when crossing large distances.

\section{REFERENCES}

[1] Hemminger, B. M., Bauers, A., and Yang, J., "Comparison of navigation techniques for large digital images," Journal of Digital Imaging (July 2008).

[2] Nacenta, M. A., Mandryk, R. L., and Gutwin, C., "Targeting across displayless space," in [Proceedings of the conference on human factors in computing systems (CHI 2008)], (April 2008).

[3] Fitts, P. M., "The information capacity of the human motor system in controlling the amplitude of movement. 1954," J Exp Psychol Gen 121, 262-269 (September 1992).

[4] Hancock, P. A. and Newell, K. M., "The movement speed-accuracy relationship in space-time.," in [Motor behaviour: Programming, Control, and acquisition], 153-188 (1985).

[5] Keyson, D. V., "Dynamic cursor gain and tactual feedback in the capture of cursor movements," Ergonomics 12, 1287-1298 (1997). 
[6] Cockburn, A. and Firth, A., "Improving the acquisition of small targets," in [HCI 2003], 181-196 (2003).

[7] Casiez, G., Vogel, D., Balakrishnan, R., and Cockburn, A., "The impact of control-display gain on user performance in pointing tasks," in [Human-Computer Interction], 23, 215 - 250 (2008).

[8] Blanch, R., Guiard, Y., and Beaudouin-Lafon, M., "Semantic pointing: improving target acquisition with control-display ratio adaptation," in [CHI '04: Proceedings of the SIGCHI conference on Human factors in computing systems], 519-526, ACM, Vienna, Austria (2004).

[9] Guiard, Y., Blanch, R., and Beaudouin-Lafon, M., "Object pointing: a complement to bitmap pointing in guis," in [GI '04: Proceedings of Graphics Interface 2004], 9-16, Canadian Human-Computer Communications Society, London, Ontario, Canada (2004).

[10] Murata, A., "Improvement of pointing time by predicting targets in pointing with a pc mouse," International Journal of Human-Computer Interaction 10, 23-32 (March 1998).

[11] A., J. M. and A., C. P., "Eye fixations and cognitive processes," Cognitive Psychology 8, 441-480 (1976).

[12] Mackenzie, I. S. and Buxton, W., "Extending fitts' law to two-dimonsional tasks," in [CHI '92: Proceedings of the SIGCHI conference on Human fctors in computing systems], 219-226, ACM, Monterey, California, United States (1992).

[13] MacKenzie, I. S., Kauppinen, T., and Silfverberg, M., "Accuracy measures for evaluating computer pointing devices," in [CHI '01: Proceedings of the SIGCHI conference on Human factors in computing systems], 9-16, ACM, Seattle, Washington, United States (2001).

[14] Tan, Y., Tien, G., Forster, B., and Atkins, M. S., "Using eyegaze targetting to improve mouse pointing for radiology tasks," in [To be presented at the Society for Imaging Informatics in Medicine SIIM 2009], (June 2009). 